\title{
Wohnwelten 2025
}

\section{Selbständigkeit und Kompetenz von Jung und Alt erhalten und fördern}

ACHIM UHL

Achim Uhl, M.Sc. leitet die

Abteilung "Ältere Menschen und Pflege« des Paritätischen Wohlfahrtsverband, Landesverband Baden-Württemberg e. V.

uhl@paritaet-bw.de

\author{
Die Entwicklung des Wohnraums wird durch \\ Veränderungen in zahlreichen Bereichen bestimmt \\ werden. Das Spektrum reicht dabei vom demografischen \\ Wandel über den sozial-gesellschaftlichen Wandel bis \\ hin zum Altersstrukturwandel, die in ihrer Gesamtheit \\ ein verändertes Nachfrageverhalten bedingen.
}

Langfristig besteht eine besondere Herausforderung in der Entwicklung angemessener Wohnformen für Menschen mit erheblichem Pflege-, Unterstützungs- und Betreuungsbedarf. Dies ist umso notwendiger, als mit dem deutlichen Rückgang der Zahl der jüngeren Menschen auch die Zahl potentieller Helfer abnimmt. Für die rasch wachsende Zahl Hochaltriger wird die Möglichkeit der "ambulanten « pflegerischen Versorgung in der eigenen Wohnung zu einem entscheidenden Kriterium der künftigen Wohnungsausstattung. In diesem Kontext kommen niedrigschwellige, wohnortnahe und quartiersbezogene Versorgungsansätze im Vorfeld von Pflege zur Sicherung des Verbleibs in der eigenen Häuslichkeit besonderer Bedeutung zu. (1)

Das Wohnen hat sich - weg von "Sonderwohnformen « - hin zur entsprechenden Ausgestaltung "normaler Wohnund Lebenswelten « entwickelt. Es steht damit ein differenziertes und flexibles Wohnangebot zur Verfügung, das auf die individuellen Lebens- und Bedürfnislagen im Lebenskontinuum ausgerichtet ist oder flexibel ausgerichtet werden kann. Wohn- und Hilfeangebote sind im normalen Wohnungsbau verankert und es steht Wohnraum für alle Lebenslagen zur Verfügung, der den Bedürfnissen von Jung und Alt gerecht wird.

Die Wohnwelten und das Umfeld haben sich derart entwickelt, dass sie die Selbständigkeit und Kompetenz von Jung und Alt erhalten und fördern. Die Wohnungs- und Siedlungsstruktu- ren sind derart gestaltet, dass sie der Bedürfnislage in jeder Lebensphase entsprechen und der sozialen Isolation entgegenwirken und die Integration ins Quartier unterstützen. Technische Hilfen im Haushalt unterstützen das selbständige Wohnen; wohnbegleitende Dienstleistungen stehen Jung und Alt zur Verfügung. Ambulante und komplementäre Versorgungsstrukturen haben zu unterschiedlichen Hilfesettings in und um die Häuslichkeit älterer Menschen geführt.

Um die wesentlichen wohnungswirtschaftlichen Dimensionen abzubilden, werden hierfür acht Themenbereiche des Wohnens (2) näher betrachtet: Haushaltsentwicklung, Ökologie, Technik, Individualisierung, Kaufkraft, Pflege, Quartiersentwicklung und Kommunikation sowie Dienstleistungen.

- Haushaltsentwicklung: Die Auflösung traditioneller familiärer Strukturen und der durch ökonomische, kulturelle und soziale Umbrüche geprägte Wandel der privaten Lebensformen und Lebensstile haben zu einem deutlichen Anstieg der Ein- und Zwei-Personen-Haushalte geführt; dieser Trend setzt sich fort, wird sich jedoch im Verlauf abschwächen. Das Nachfrageverhalten und der Bedarf in diesem Kontext wird heterogener: In zunehmendem Maße werden auf der einen Seite größere Wohnungen nachgefragt, jedoch wächst auf der anderen Seite die Nachfrage und der Bedarf von kleinen preisgünstigen 
Wohnungen. Zunehmend bestimmen die »Neuen Alten «, deren Bedürfnisse und Lebensformen sich stark ausdifferenziert haben, neue Wohnkonzepte. Familiäre Hilfepotentiale sind aufgrund der Singularisierung (erheblichen Anteil von Ein-Kind- und KeinKind-Familien und Scheidungen) begrenzt. Die Wohnformen ziehen somit differenzierte Nachfragestrukturen nach sich, die spezifische Anforderungen an Wohnen und Dienstleistungen stellen. Neue Lebensformen, geprägt von Alleinerziehenden, nichtehelichen Lebensgemeinschaften und anderen Patchwork-Strukturen, sind hinzugekommen und erweitern die Anforderungen an familiengerechten Wohnen.

- Wohnökologie: Wohnökologie ist mittlerweile in breiten Schichten der Bevölkerung etabliert und wird das Nachfrageverhalten beeinflussen. »Es besteht daher auf der Angebotsseite ein zunehmender Bedarf an intelligenten Gesamtsystemen aus entsprechendem Gebäudedesign und energieeffizienter Haustechnik (>Green Buildings $<$ ). (3) Konzepte, wie zum Beispiel »Smart Metering « ermöglichen, dass der Nutzer genau feststellen kann, welches Verhalten und welche Geräte wie hohe Verbräuche und Kosten verursachen. Dies wird insbesondere für Milieus mit niedrigem Einkommen an Bedeutung gewinnen. (4)

- SMART Home: Die Akzeptanz für technische Lösungen in der Wohnausstattung, die unabhängig vom Alter der Nutzer ist, steigt. Im Vordergrund stehen dabei technische Lösungen (5), die mehr Komfort, mehr Sicherheit, eine bessere Wirtschaftlichkeit und eine Reduzierung des individuellen Energieverbrauches im Wohnumfeld ermöglichen. "Smart-Home-Technologien " (6) und netzwerkgestützte Serviceleistungen bei der Wohnungsausstattung tragen zu einer Erhöhung von Sicherheit, Komfort und Gesundheit des Nutzers bei. Selbständigkeit, Kommunikation und die Mobilität können durch technische Geräte unterstützt und bei nachlassenden Fähigkeiten kompensiert werden.

- Neue Werte, anderes Wohnen: Die gesamtgesellschaftlichen Entwicklungsprozesse der Individualisierung und Pluralisierung verändern die Sozialstruktur in den kommenden Jahren. Die Wohnung wird zum Ort der
Selbstverwirklichung und Kulisse für die Selbstdarstellung. (7) Die zentrale sozialgesellschaftliche Entwicklung ist der Trend hin zur Individualisierung, die ihren Ausdruck in der Ausgestaltung der Wohnung als auch hinsichtlich der Merkmale des Wohnungsangebotes findet. Hierbei sind drei zentrale Prinzipien von Bedeutung. Erstens: "Leben in Privatheit« (8): Hier stehen Sicherung und Abgrenzung der Privatsphäre mit stärkerer Betonung des Rückzugsraumes im Vordergrund, aber auch Gemütlichkeit. Zweitens: "Leben in Gemeinschaft«: Wesentlich ist hier, dass "Gemeinschaftlichkeit« (9) - Leben in familienähnlichen Strukturen (10) - durch gemeinsames Erleben im (halb-) öffentlichen Raum entsteht, während die Privatsphäre davon überwiegend frei gehalten wird. Formen wie Mehrgenerationenhäuser, (Senioren-) Wohngemeinschaften und Baugemeinschaften kommen zwar dem wachsenden Bedürfnis an institutionalisierter Gemeinsamkeit nach, bleiben aber Nischenprodukte für eine kleine Gruppe, insbesondere für die Haushalte mit häuslich-familiären Wohnkonzepten. (11) Drittens: »Leben in der Öffentlichkeit«: Ein sozialraumorientierter Ansatz zur Versorgung unterstützt die Selbsthilfepotentiale der Bewohner und vermittelt Sicherheit und Lebensqualität durch eine bedarfsgerechte Unterstützung im Quartier. Um den aktuellen und den zukünftig zu erwartenden Anforderungen an den WohnRaum gerecht zu werden, ist es notwendig, sich von einer monofunktionalen Zuordnung von Raum und Funktion abzuwenden. Erforderlich ist ein Ansatz der Wohnraumgestaltung, der nicht von Räumen ausgeht, sondern von funktionalen Zonen, die innerhalb einer Wohnung flexibel vorhanden sein sollten.

- Kaufkraft: Die ökonomische Basis der Privathaushalte insbesondere von Haushalten mit Niedrigeinkommen (Arbeitslosenhaushalte, Alleinerziehende und Haushalte mit Migrationshintergrund) nach preiswerten Wohnungen wird steigen. Der soziale Wohnungsbau wird zukünftig eine bedeutendere Rolle spielen, da die Nachfrage nach preiswertem Wohnraum durch den regulierenden Eingriff bei der Übernahme der Kosten der Unterkunft bei Einkommensschwachen steigen wird.

- Wohnen und Pflege: Dem Wohnen, insbesondere dem Wohnen im Alter, wird zukünftig in zunehmendem Maße erweiterte Funktionen im Hinblick auf Größe und technische Ausstattung der Wohnung, als auch hinsichtlich des Angebots und der Ausgestaltung von Service und Pflegeleistungen zukommen. Moderne Informations- und Kommunikationsmittel (12) ermöglichen in immer größerem Umfang älteren Menschen

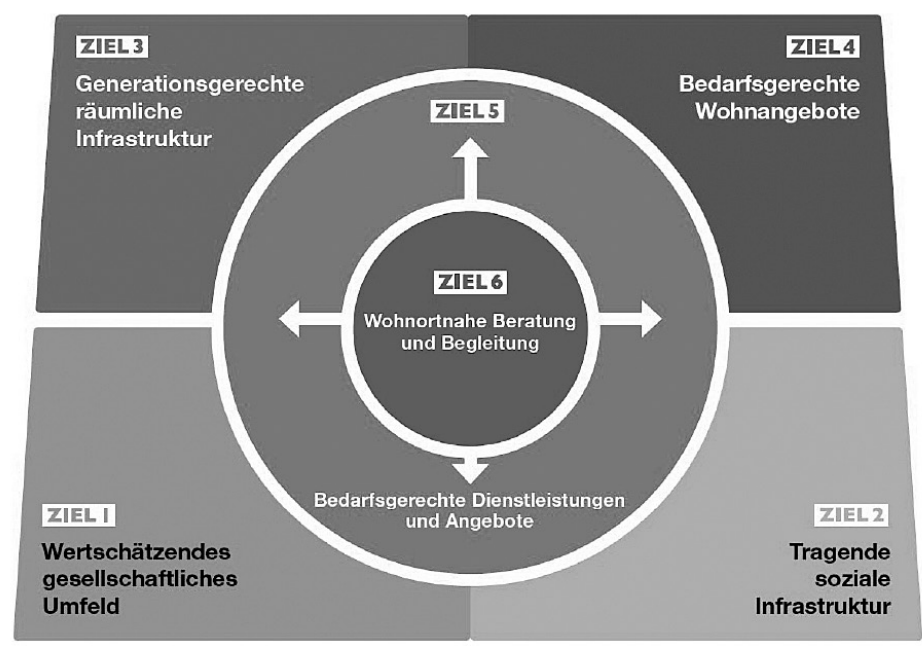

Abb. 1: Angesichts des demografischen und sozialgesellschaftlichen Wandels müssen die Strukturen vor Ort durch Quartierskonzepte nach verschiedenen Zielen weiterentwickelt werden.

Quelle: KDA 2011 
in ihren angestammten Wohn- und Lebenszusammenhängen, in der eigenen Häuslichkeit zu verbeiben. Die Wohnung wird zukünftig zunehmend zu einem Ort des Pflegens. Das Ziel integrierter »intelligenter « Systeme liegt dabei in dem Angebot von Leistungen, die auf die individuellen Bedürfnisse des Nutzers im (angepassten) Wohnumfeld ausgerichtet sind.

- Quartiersentwicklung und Kommunikation: Um den demografischen und sozialgesellschaftlichen Wandel zu begegnen, müssen die Strukturen vor Ort durch Quartierskonzepte weiterentwickelt werden. Das Kuratorium Deutsche Altershilfe hat ein Zielsystem (13) entwickelt, das wesentliche Aspekte der Quartiersentwicklung beschreibt (vgl. Abb. 1): Ziel 1: Wertschätzendes gesellschaftliches Umfeld: Bürgerinnen und Bürger übernehmen füreinander im Quartier Verantwortung. Ziel 2: Tragende soziale Infrastruktur: Aufbau funktionierender Sozialbeziehungen, die im Sinne der Nachbarschaftsarbeit zu sozialen Netzwerken ausgebaut und nachbarschaftliche Hilfeleistungen ermöglichen. Ziel 3: Generationsgerechte räumliche Infrastruktur: Von Bedeu- tung sind hier barrierefreie öffentliche Räume und Gebäude, Bereitstellung von Erholungs- und Freizeitmöglichkeiten im Umfeld. Ziel 4: Bedarfsgerechte Wohnangebote: Der »normale« Wohnbestand ermöglicht Menschen mit Mobilitäts-, Sinnes- oder Orientierungseinschränkungen den Verbleib in der Häuslichkeit. Ziel 5: Bedarfsgerechte Dienstleistungen und Angebote: Ein (längerer) Verbleib im Quartier setzt die Verfügbarkeit von bedarfsgerechten professionellen und ehrenamtlichen Dienstleistungen und Angeboten zur Hilfe- und Unterstützung voraus. Ziel 6: Wohnortnahe Beratung und Begleitung: Anlaufstelle für Bürgerinnen und Bürger des Quartiers mit der Funktion des Case- und CareManagements. Bedingt durch die sozialgesellschaftlichen Prozesse haben sich die sozialen Netzwerke verändert: Direkte Kontakte haben an Bedeutung verloren und werden durch territoriale Bezüge zunehmend aufgelöst und durch personenbezogene, großräumigere Netze (Virtualisierung) ersetzt. Die Quartiersentwicklung verfolgt in diesem Kontext den Aufbau und den Erhalt von funktionierenden nachbarschaftlichen Kontakten. Der Aspekt

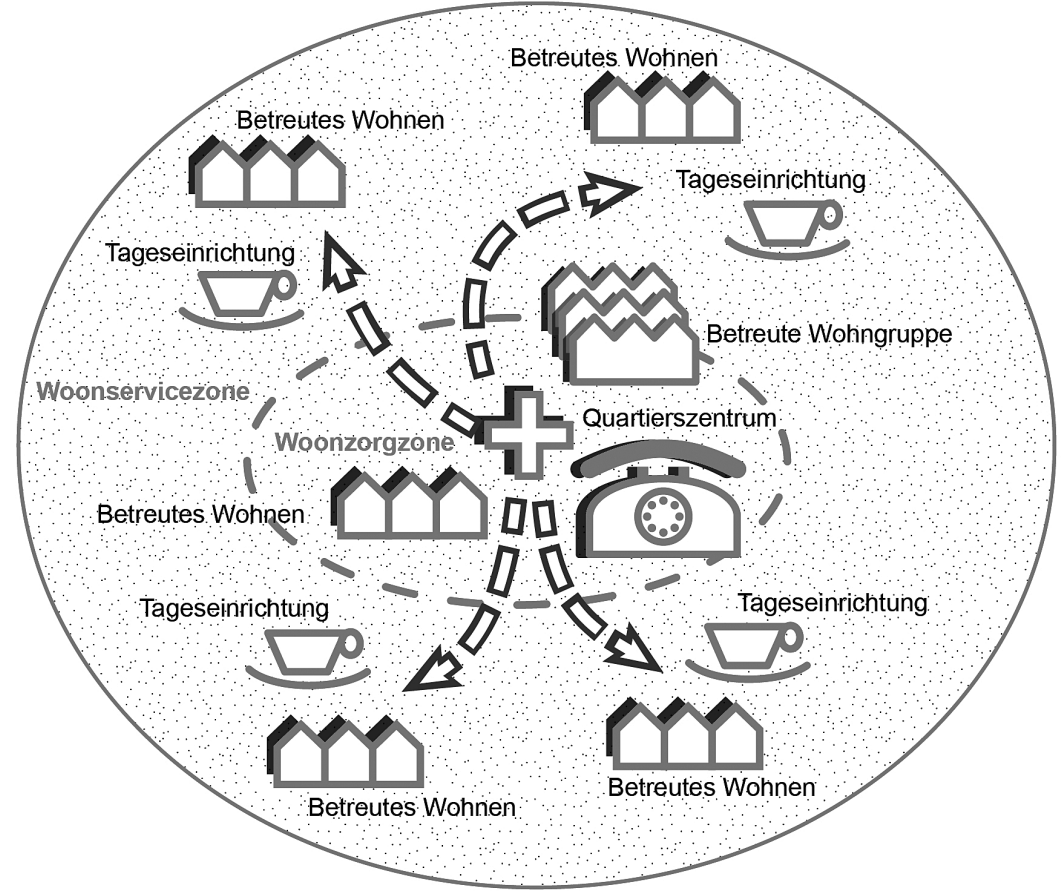

Abb. 2: Das in den Niederlanden entwickelte STAGG-Modell der betreuten Wohnzone ist besonders auf das Leben in einem Stadtquartier ausgerichtet.

Quelle: Eigene Darstellung, Grundlage: Singelenberg 2010: 10. des Quartiersmanagements wird einen zentralen Stellenwert erhalten, um dem Bedürfnis nach direkter Kommunikation, gegenseitiger Unterstützung und Aktivität im Quartier nachzukommen. Funktionierende aktive Nachbarschaften sind insbesondere für Senioren von Bedeutung, die in der Mobilität eingeschränkt sind.

- Dienstleitungen: Die Bereitschaft, wohnbegleitende Dienstleistungen in Anspruch zu nehmen, steigt. Individuelle Angebote, die sich gezielt an Jüngere (Zeitersparnis) oder Ältere (mangelnde Fähigkeiten, die Aufgaben selbst zu erledigen) wenden, stellen zentrale Dienstleistungen dar. In Zukunft kommt es zu einer steigenden Nachfrage nach altersgerechten Wohnungen, nach alternativen Wohnformen mit haushaltsnahen Dienstleistungen als auch nach Wohnformen mit umfassender pflegerischer Betreuung, die es älteren Menschen ermöglichen, auch bis ins hohe Alter in der eigenen Wohnung angemessen versorgt zu werden. In diesem Kontext ist ebenfalls das Quartiersmanagement zu nennen, das den Fokus auf die Aufrechterhaltung der sozialen Kontakte auf Quartiersebene legt und Bewegungsmöglichkeiten schafft, sowie Kontakte zwischen den Bewohnern initiiert.

\section{Das STAGG-Modell der Quartiersentwicklung}

Das STAGG-Modell geht auf eine Veröffentlichung der niederländischen Stiftung Architektenforschung für Gebäude und Gesundheitswesen (Stichting Architectenonderzoek Gebouwen Gezondheidszorg STAGG) aus dem Jahr 1995 zurück und definiert eine Basishierarchie von Wohnen und Pflegeeinrichtungen für ein Quartier mit 5.000 bis 10.000 Einwohnern. (14) Innovativ ist die Aufteilung der bis dahin in stationären Einrichtungen erbrachten Leistungen in die einzelnen Bausteine Wohnen, Pflege und sonstige (gesundheitsbezogene) Dienstleistungen. Zur räumlichen Verankerung der Funktionen wird ein Quartier nach dem STAGG-Modell in zwei Zonen mit unterschiedlichen Funktionen und Ausstattungen aufgeteilt (vgl. Abb. 2).

Die »Woonzorgzone" (Wohnpflegezone) stellt das Zentrum einer betreu- 
ten Wohnzone dar. In der Mitte dieses Gebiets liegt für alle gut erreichbar ein Quartierszentrum, das vier Funktionen in einem integrativen Ansatz vereint (15). Idealerweise befindet sich das Quartierszentrum in der Nähe der örtlichen Einzelhandelsgeschäfte, so dass alle wichtigen Einrichtungen des Viertels an einem zentralen, fußläufig gut erreichbaren Standort liegen. Die eigentliche Woonzorgzone erstreckt sich in einem Radius von ca. 300 Metern (entspricht ca. 10 Minuten Fußweg mit einem Rollator) um das Quartierszentrum. In dieser Zone befinden sich mehrere kleinteilige Pflegehäuser, die von Pflegekräften des Quartierszentrums versorgt werden (16).

Das STAGG-Modell unterscheidet zwei Arten von Pflegehäusern: »Betreutes Wohnen« und »Betreute Wohngruppen«. Bewohner des Betreuten Wohnens leben in einer eigenen Wohnung im Pflegehaus, nutzen aber zur Aufrechterhaltung des eigenen Haushalts Hilfsangebote und bei Bedarf ambulante Pflegeangebote des Quartierszentrums. In den Betreuten Wohngruppen leben pflegebedürftige Menschen mit 24-Stunden-Betreuung. Es kann sich dabei um Gruppen von Senioren oder behinderten Menschen handeln. Neben den Gemeinschaftsräumen haben die Bewohner der Wohngemeinschaft auch einen privaten (Schlaf-) Bereich. Darüber hinaus soll innerhalb der Woonzorgzone eine möglichst hohe Anzahl barrierefreier Wohnungen im regulären Bestand verwirklicht werden, damit so viele Menschen mit Unterstützungsbedarf wie möglich in der Nähe des Quartierszentrums wohnen können. Der Anteil der Wohnungen in den eigentlichen Pflegehäusern sollte 25 Prozent des örtlichen Bestandes nicht überschreiten, um die Atmosphäre eines normalen Wohnviertels nicht zu gefährden. Über ein barrierefreies Wohnumfeld und Wegenetze werden die zentralen Einrichtungen des Quartiers, insbesondere das Quartierszentrum, für alle Bewohner gut erreichbar gestaltet.

Das übrige Gebiet des Quartiers (außerhalb der Woonzorgzone) wird als Woonservicezone (Wohnservicezone) bezeichnet. Über das Pflegeteam des Quartierszentrums können auch in der Woonservicezone ambulante Pflegeleistungen erbracht werden. Darüber hinaus existiert ein dichtes Netz haus- haltsnaher Dienstleistungen und Hilfsangebote, die zum möglichst langen selbständigen Wohnen in der eigenen Häuslichkeit beitragen. Innerhalb der Woonservicezone sind als dezentrale Ergänzung zum Quartierszentrum der Woonzorgzone kleinere Tageseinrichtungen vorgesehen, die fußläufig gut erreichbare Angebote für Tagesaktivitäten anbieten. Baulich zeichnet sich die Woonservicezone durch einen möglichst großen Bestand an barrierefreien Wohnungen und (analog zur Woonzorgzone) ein ebenfalls barrierefreies und sicheres Wohnumfeld für Fußgänger und Radfahrer aus. (17)

\section{Anmerkungen}

(1) Uhl 2012: 17.

(2) Vgl. GdW 2008, angelehnt.

(3) Vgl. GdW 2008.

(4) Vgl. GdW 2008.

(5) Z. B. automatische Heizungssteuerung, sensorgesteuerte Türen, Lichter und Rollläden, »intelligente « Kühlschränke, die niemals leer sind.

(6) Technische Hilfen lassen sich grob in drei Bereiche einteilen: Sicherheits-Meldesysteme, Steuerungssysteme, Unterstützungssysteme.

(7) Vgl. GdW 2008.

(8) Vgl. KDA 2012; GdW 2008.

(9) Gemeinschaftlichkeit in sozialer und ökonomischer Hinsicht spielt eine wichtige Rolle.

(10) Vgl. KDA 2012; GdW 2008.

(11) Vgl. GdW 2008.

(12) Z. B. eHealth-Geschäftsmodelle, TeleHomeCare und »intelligente « Gesundheitsportale.

(13) Vgl. KDA 2011.

(14) Vgl. Singelenberg 2010.

(15) Vgl. Singelenberg 2010.

(16) Vgl. Singelenberg 2010.

(17) Vgl. Singelenberg 2010.

\section{Literatur}

Bucher, Hansjörg \& Claus Schlömer (2008):

Räumliche Varianz des demographischen

Wandels in Deutschland. In: Geographie und

Schule, 30 (172): 11-16

Bundesministerium für Familie, Senioren,

Frauen und Jugend (1998): Wohnen im Alter.

Zweiter Altenbericht. Bonn.

Bundesverband deutscher Wohnungs- und Immobilienunternehmen e.V. (GdW) (Hg.)

(2008): Wohntrends 2020. Studie. Wohnkon- zepte, Struktur und Wohnkaufkraft der Haushalte in Deutschland. Ein Modell für die Praxis. Branchenbericht 3. Hamburg.

Dangschat, Jens S. \& Hamedinger, Alexander (Hg.)(2007): Lebensstile, soziale Lagen und Siedlungsstrukturen. Forschungs- und Sitzungsberichte der ARL. Hannover. Band 230. Gans, Paul \& Schmitz-Veltin, Ansgar (2008): Demographischer Wandel. Ein globaler Prozess? In: Geographie und Schule, 30 (172): 4-11. Kemper, Franz-Josef (2006): Komponenten des demographischen Wandels und die räumliche Perspektive. In: Raumforschung und Raumordnung, 64 (3): 195-199.

Kuratorium Deutsche Altenhilfe (KDA) (2011): Quartiersentwicklung. Ziele, Verantwortlichkeiten und politischer Handlungsbedarf. Köln, www.kda.de/tl files/kda/PDF-Dateien/2011KDA-Michell-Auli-Quartiersentwicklung.pdf, aufgerufen am 29.07.2012.

Kuratorium Deutsche Altenhilfe (KDA) (2012):

Die 5. Generation: KDA-Quartiershäußer.

Ansätze zur Neuausrichtung von Alten- und

Pflegeheimen. Band 6. Köln.

Rohr-Zänker, Ruth \& Müller, Wolfgang (1989): Die Rolle von Nachbarschaften für die zukünftige Entwicklung von Stadtquartieren. Expertise im Auftrag der Bundesforschungsanstalt für Landeskunde und Raumordnung. Oldenburg. Singelenberg, Jeroen (2010): Nationaler Kontext: Niederlande. Papier der ISAPlattform (Integrated Service Areas). Köln: ISA, www.isaplatform.eu/uploads/media/Niederlande.pdf, aufgerufen am 29.07.2012.

Sinus Markt- und Sozialforschung (2011): Informationen zu den Sinus-Milieus 2011. Heidelberg.

Sinus Sociovision (2007): Informationen zu den Sinus-Milieus 2007. Heidelberg.

Statistisches Bundesamt (2009): Bevölkerung Deutschlands bis 2060, 12. koordinierte Bevölkerungsvorausberechnung. Wiesbaden. Statistisches Bundesamt (2011): Bevölkerung und Erwerbstätigkeit, Vorläufige Ergebnisse der Bevölkerungsfortschreibung 2009. Wiesbaden.

- Tews, Hans-Peter (1993): Neue und alte Aspekte des Strukturwandels des Alters. In: Naegele, Gerhard \& Hans-Peter Tews (Hg.): Lebenslagen im Strukturwandel des Alters. Opladen: 15-42. Uhl, Achim (2012): Die Zukunft altersgerechter (Wohn-)Quartiere gestalten. In: Der Paritätische Baden-Württemberg und Wüstenrot Stiftung (2012) (Hg.): Älter werden im Quartier. Neue Netzwerke - Aktive Teilhabe - Mehr Versorgungssicherheit. Ludwigsburg. Weymann, Ansgar (1998): Sozialer Wandel. Theorien zur Dynamik der modernen Gesellschaft, Juventa, Weinheim/München. 